\title{
3-Methylcholanthrene Induces Chylous Ascites in TCDD-Inducible Poly-ADP-Ribose Polymerase (Tiparp) Knockout Mice
}

\author{
Tiffany E. Cho ${ }^{1}$, Debbie Bott ${ }^{1}$, Shaimaa Ahmed ${ }^{1}$, David Hutin ${ }^{1}$, Alvin Gomez ${ }^{1}$, \\ Laura Tamblyn ${ }^{1}$, Angela C. Zhou ${ }^{2}$, Tania H. Watts ${ }^{2}$, Denis M. Grant ${ }^{1}$ and Jason Matthews ${ }^{1,3, *(D)}$ \\ 1 Department of Pharmacology and Toxicology, University of Toronto, Toronto, ON M5S 1A8, Canada; \\ tiffany.cho@mail.utoronto.ca (T.E.C.); debb.bott@gmail.com (D.B.); shaimaa.ahmed1@gmail.com (S.A.); \\ dhutin1@gmail.com (D.H.); alvin_v_gomez@yahoo.com (A.G.); Laura.Tamblyn@uhnresearch.ca (L.T.); \\ denis.grant@utoronto.ca (D.M.G.) \\ 2 Department of Immunology, University of Toronto, Toronto, ON M5S 1A8, Canada; \\ angel.zhou@mail.utoronto.ca (A.C.Z.); tania.watts@utoronto.ca (T.H.W.) \\ 3 Department of Nutrition, Institute of Basic Medical Sciences, University of Oslo, Sognsvannsveien 9 , \\ 0372 Oslo, Norway \\ * Correspondence: Jason.matthews@medisin.uio.no
}

Received: 16 April 2019; Accepted: 7 May 2019; Published: 10 May 2019

\begin{abstract}
TCDD-inducible poly-ADP-ribose polymerase (TIPARP) is an aryl hydrocarbon receptor (AHR) target gene that functions as part of a negative feedback loop to repress AHR activity. Tiparp /- $^{-}$ mice exhibit increased sensitivity to the toxicological effects of 2,3,7,8-tetrachlorodibenzo-p-dioxin (TCDD), including lethal wasting syndrome. However, it is not known whether Tiparp ${ }^{-1-}$ mice also exhibit increased sensitivity to other AHR ligands. In this study, we treated male Tiparp ${ }^{-1-}$ or wild type (WT) mice with a single injection of $100 \mathrm{mg} / \mathrm{kg}$ 3-methylcholanthrene (3MC). Consistent with TIPARP's role as a repressor of AHR signaling, 3MC-treated Tiparp $^{-/-}$mice exhibited increased hepatic Cyp1a1 and Cyp1b1 levels compared with WT mice. No 3MC-treated Tiparp ${ }^{-/-}$mice survived beyond day 16 and the mice exhibited chylous ascites characterized by an accumulation of fluid in the peritoneal cavity. All WT mice survived the 30-day treatment and showed no signs of fluid accumulation. Treated Tiparp $^{-1-}$ mice also exhibited a transient and mild hepatotoxicity with inflammation. 3MC-treated WT, but not Tiparp ${ }^{-/-}$mice, developed mild hepatic steatosis. Lipid deposits accumulated on the surface of the liver and other abdominal organs in the 3MC-Tiparp ${ }^{-/}$mice. Our study reveals that Tiparp ${ }^{-/}$ mice have increased sensitivity to 3MC-induced liver toxicity, but unlike with TCDD, lethality is due to chylous ascites rather than wasting syndrome.
\end{abstract}

Keywords: TCDD-inducible poly-ADP-ribose polymerase (TIPARP); 3-methylcholanthrene; chylous ascites; wasting syndrome; 2,3,7,8-tetrachlorodibenzo- $p$-dioxin

\section{Introduction}

The aryl hydrocarbon receptor (AHR) is a ligand-dependent transcription factor that mediates a wide range of biological effects in response to endogenous and dietary ligands. Toxicological effects are induced upon activation by numerous environmental and synthetic ligands, including 2,3,7,8-tetrachlorodibenzo- $p$-dioxin (TCDD) and polycyclic aromatic hydrocarbons (PAHs) such as 3-methylcholanthrene (3MC) [1]. In response to 3MC or other AHR agonists, the AHR translocates to the nucleus where it heterodimerizes with AHR nuclear translocator (ARNT) and the complex then binds to AHR response elements (AHREs) located in the 5' regulatory region of hundreds of genes, 
including cytochrome P4501A1 (CYP1A1), CYP1B1, and TCDD-inducible poly-ADP-ribose-polymerase (TIPARP) [1,2].

PAHs are a group of ubiquitous environmental pollutants that are generated from anthropogenic and natural incomplete combustion processes [3]. Important sources include cigarette smoke, diesel exhaust, and grilled foods [4]. The main routes of exposure for humans are via the inhalation of PAHs from the ambient air and through the ingestion of charred foods [5]. The toxic mechanism of action for PAHs includes both their well-established genotoxicity, which results from their bioactivation to mutagenic metabolites that form DNA adducts, and their ability to mediate non-genotoxic effects through various intracellular receptors [6,7]. The metabolism of PAHs is multifaceted with the AHR-mediated induction of drug-metabolizing enzymes, such as CYP1A1 and CYP1B1, being central to the generation of reactive metabolites [3]. 3MC is a synthetic PAH that is genotoxic and mutagenic, and has also been reported to increase cell proliferation, alter reproduction, modulate estrogen signaling, and activate the AHR [8-10]. However, unlike TCDD, a single high-dose exposure to 3MC does not cause severe hepatotoxicity or induce wasting syndrome, although mild hepatosteatosis has been reported [11].

TIPARP, also known as poly-ADP-ribose polymerase 7 (PARP7) or ADP-ribosyltransferase diphtheria toxin-like 14 (ARTD14), is an AHR target gene and a member of the PARP family of proteins, which catalyze the transfer of ADP-ribose units onto themselves or onto acceptor proteins [12-14]. ADP-ribosylation is a post-translational modification that is involved in numerous cellular processes, including metabolism, DNA repair, immune cell function and regulation, protein stability, and gene regulation [15]. TIPARP is a mono-ADP-ribosyltransferase and it is predominantly localized in the nucleus in many cell types [12,16]. Its cellular localization is dependent on a N-terminal nuclear localization signal and a CCCH-zinc finger domain [17]. TIPARP is involved in several biological processes including innate immunity, responses to viral infection, stem cell pluripotency, astrocyte autophagy, and the regulation of transcription [12,18-20]. TIPARP expression is induced by platelet-derived growth factors [21], viral infection [22], nuclear hormone receptors [23] and AHR [2]. Increasing evidence suggests that TIPARP functions as part of a negative feedback loop to regulate AHR signaling through mono-ADP-ribosylation $[12,13]$. Tiparp ${ }^{-1-}$ and hepatocyte-specific Tiparp ${ }^{-1-}$ mice treated with a normally non-lethal dose of $10 \mu \mathrm{g} / \mathrm{kg}$ TCDD exhibit increased sensitivity to TCDD-induced toxicities including the development of steatohepatitis, hepatotoxicity, and lethal wasting syndrome $[13,24]$. However, whether Tiparp ${ }^{-/}$mice exhibit increased sensitivity to other AHR ligands has not been determined.

In the present study, we treated male Tiparp ${ }^{-/-}$or wild type (WT) mice with a single non-lethal dose of $100 \mathrm{mg} / \mathrm{kg} 3 \mathrm{MC}$ and monitored them for up to 30 days. Interestingly, no 3MC-treated Tiparp ${ }^{-/-}$ mice survived beyond day 16 . This increased sensitivity to $3 \mathrm{MC}$-induced lethality was not due to severe hepatotoxicity or wasting syndrome, but rather the mice exhibited a chylous ascites condition characterized by the peritoneal accumulation of a viscous fluid with high lipid and protein content. Our data show that TIPARP has an important role in modulating the differential toxic effects of two distinct AHR ligands, and further characterize it as a key regulator of the AHR signaling pathway.

\section{Results}

In a previous study by our group, we reported that treatment of male or female Tiparp $^{-/-}$mice with a single intraperitoneal (IP) injection of $100 \mu \mathrm{g} / \mathrm{kg}$ TCDD resulted in an enhanced sensitivity to wasting syndrome causing death between day 3 and 5; treated WT mice survived to the end of the 30-day period [13]. These data provided further support for TIPARP's role as a negative regulator of AHR activity [12]. To determine if Tiparp ${ }^{-/-}$mice exhibit increased AHR signaling in the presence of another, but readily metabolizable AHR ligand, male Tiparp $^{-/-}$mice and WT mice were treated with a single IP injection of $100 \mathrm{mg} / \mathrm{kg}$ 3MC. The hepatic mRNA expression levels of Cyp1a1 and Cyp1b1 were significantly higher in 3MC-treated Tiparp $^{-/-}$mice compared with WT mice after a $6 \mathrm{~h}$ exposure (Figure 1A,B). Tiparp mRNA levels were increased in WT but not in Tiparp-/- mice (Figure 1C). 
A

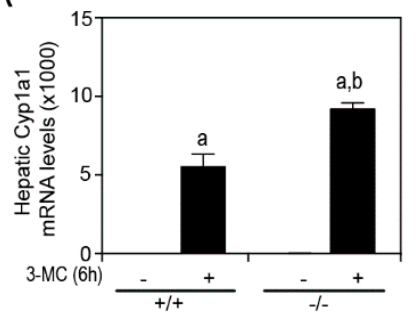

B

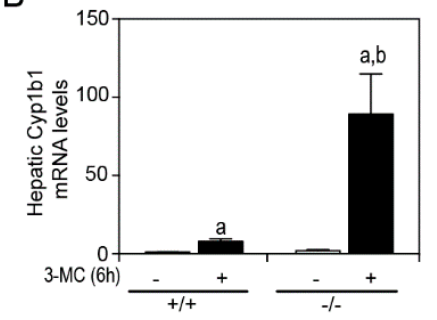

C

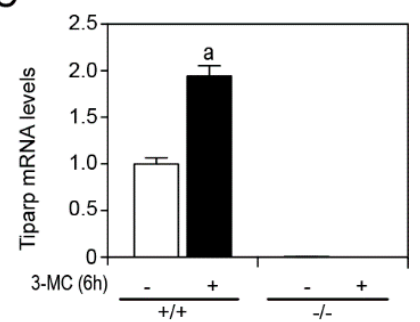

Figure 1. Hepatic gene expression of aryl hydrocarbon receptor (AHR) target genes in male mice $6 \mathrm{~h}$ after treatment with $100 \mathrm{mg} / \mathrm{kg}$ 3MC. Hepatic RNA was isolated, reverse transcribed and mRNA expression levels of (A) Cyp1a1, (B) Cyp1b1 and (C) Tiparp were determined by qPCR. Data represent the mean \pm $\mathrm{SEM} ; n=3$ for all genes. ${ }^{\mathrm{a}} p<0.05$ two-way ANOVA comparison between genotype-matched corn oiland 3MC-treated mice and ${ }^{\mathrm{b}} p<0.05$ two-way ANOVA comparison between treatment-matched WT and Tiparp $^{-/-}$mice followed by a Tukey's post hoc test.

To study if Tiparp $^{-/-}$mice exhibit increased sensitivity to 3MC-induced toxicity, we treated Tiparp $^{-/-}$and WT mice with a single IP injection of $100 \mathrm{mg} / \mathrm{kg} 3 \mathrm{MC}$ and monitored them for up to 30 days. All WT mice treated with 3MC survived the duration of the study without any signs of distress. In contrast, the 3MC-treated Tiparp ${ }^{-/}$mice died on or between days 8 to 16 (Figure 2A). The mice were either found dead in the morning on the day of death or had to be humanely euthanized due to poor health. An initial decrease in body weight of 3MC-treated Tiparp ${ }^{-1-}$ mice was followed by an increase after day 8. No significant differences in food intake were observed. This was most likely due to high variability among the animals and the lower number of surviving Tiparp ${ }^{-1-}$ mice as the 30-day experiment progressed. (Figure 2B,C). In each of the 3MC-treated Tiparp ${ }^{-1-}$ mice, abdominal distention was evident around days 5-6. This distention was due to the accumulation of fluid in the peritoneum, which may have accounted for the gradual increase in weight over time. All fluid samples obtained from these mice were milky white and viscous (Figure 2D).

We conducted a subsequent acute 6-day toxicity study to characterize the effects of 3MC on the Tiparp $^{-1-}$ mice. Similar to that observed in the 30-day study, a translucent to milky white and viscous fluid accumulated in the peritoneal cavities of all 3MC-treated Tiparp ${ }^{-1}$ mice on day 6 (Figure 3A). However, the samples were less viscous and more translucent compared with treated Tiparp ${ }^{-1}$ mice in the 30-day study. Biochemical analyses of the collected fluid samples from the 3MC-treated Tiparp-/mice in the 30-day and 6-day study revealed high triglyceride levels and high protein concentrations. The triglyceride levels of the viscous fluid ranged from 322 to $1923 \mathrm{mg} / \mathrm{dL}$, while protein levels ranged from 1.9 to $2.6 \mathrm{~g} / \mathrm{dL}$ (Table 1). Wright Giemsa staining of the fluid revealed a high concentration of immune cells (Figure 3B). Flow cytometry was then used to phenotype the cells. The majority of the cells were hematopoietic (CD45.2+) and they were predominantly innate cell types since they were negative for CD3 and CD19, which are markers for T and B cells, respectively. These innate cells were not antigen-presenting cells (CD11c-, MHC II-) but were predominantly neutrophils (Ly6C+, Ly6G+) (Figure 3C). Based on these biochemical parameters, the fluid was similar to chylous ascites in humans [25], except for the predominance of neutrophils rather than the characteristic lymphocytic population observed in patients (Table 1). 
A

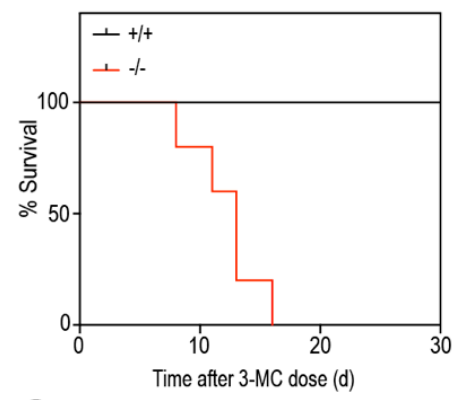

C

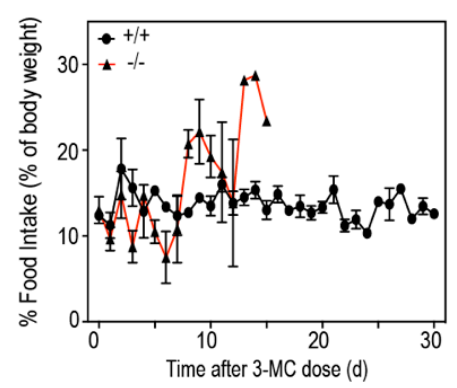

B

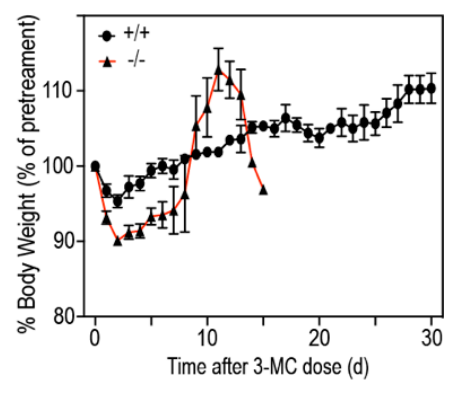

D

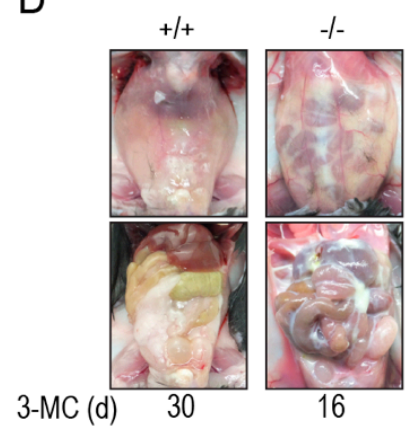

Figure 2. Thirty-day survival study characteristics. (A) Kaplan-Meier survival curves indicating the survival rate of 3MC-treated WT (+/+) and Tiparp ${ }^{-/-}(-/-)$mice. Mice were euthanized when body weight loss exceeded $20 \%$ of the baseline (day 0 ) value or if the animal had reached an endpoint as described in the Materials and Methods. (B) Daily body weights expressed as a percent of baseline values (i.e., day 0). (C) Daily food intake measurements expressed as a gram per gram percentage of daily mouse body weight normalized to baseline values. For B and C, the data represent the mean \pm SEM; $n=4-6$. (D) Representative images of the closed peritoneum and opened peritoneal cavity of WT (left; day 30) and Tiparp ${ }^{-/}$mice (right; day 16). Images on the top show an intact peritoneum with fluid accumulation in the 3MC-treated Tiparp ${ }^{-1-}$ mice (right) compared with similarly treated WT mice (left). Images on the bottom show the open abdomen and all tissues in the peritoneal cavity. Reduced epididymal white adipose tissue was observed in the Tiparp ${ }^{-/}$mice with remnants of the fluid adhering to the tissues.

Table 1. Characteristics of chylous ascites observed from 3MC-treated Tiparp ${ }^{-1-}$ mice. Values for the clinical diagnosis of chylous ascites were adapted from Cárdenas and Chopra (2002) [25]. Values in parentheses indicate the number of observations over the number of total observations.

\begin{tabular}{|c|c|c|c|}
\hline Measure & $\begin{array}{l}\text { Clinical Diagnosis of } \\
\text { Chylous Ascites }\end{array}$ & Tiparp ${ }^{-/-}$Mice Days 8-16 & Tiparp ${ }^{-/-}$Mice Day 6 \\
\hline Appearance & Milky white and cloudy & Milky white and cloudy (7/7) & $\begin{array}{l}\text { Milky white and cloudy }(4 / 6) \\
\text { White and translucent }(2 / 6)\end{array}$ \\
\hline Triglyceride Level & $>200 \mathrm{mg} / \mathrm{dL}$ & 48-2065 mg/dL & $322-1923 \mathrm{mg} / \mathrm{dL}$ \\
\hline Cell Population & Lymphocytes & Neutrophils & Neutrophils \\
\hline Total Protein & $1.1-7.0 \mathrm{~g} / \mathrm{dL}$ & $1.9-5.1 \mathrm{~g} / \mathrm{dL}$ (mean: $3.8 \mathrm{~g} / \mathrm{dL}$ ) & $1.9-2.6 \mathrm{~g} / \mathrm{dL}$ (mean: $2.2 \mathrm{~g} / \mathrm{dL}$ ) \\
\hline
\end{tabular}

Since liver toxicity is a contributing factor to chylous ascites, we examined the livers of 3MCand corn oil treatments in WT and Tiparp ${ }^{-1-}$ mice. Significant reductions in body weight for both treated WT and Tiparp ${ }^{-1-}$ mice were observed at day 3, but only for Tiparp ${ }^{-1-}$ mice at day 6 (Figure 4A). Both 3MC-treated WT and Tiparp ${ }^{-1-}$ mice had increased liver weights (Figure 4B). 3MC-treated Tiparp $^{-/-}$mice had a significant, but transient, increase in serum alanine aminotransferase (ALT) activity (Figure 4C) on day 3, which returned to baseline on day 6 . We next determined the hepatic AHR-regulated gene expression after $6 \mathrm{~h}$ and at day 6. The mRNA levels of Cypla1 were not 
significantly different between genotypes; however, Cyp1b1 mRNA levels were significantly greater in 3MC-treated Tiparp ${ }^{-/}$mice compared with similarly treated WT mice (Figure 4D,E).

A

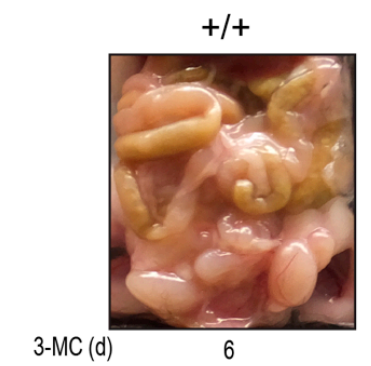

C

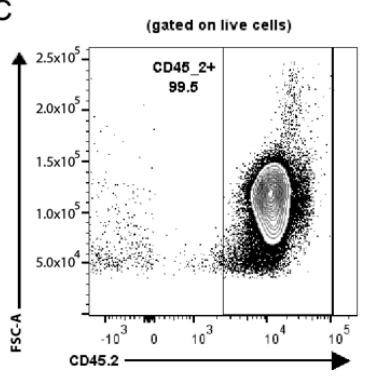

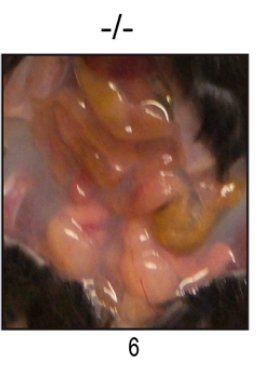

B
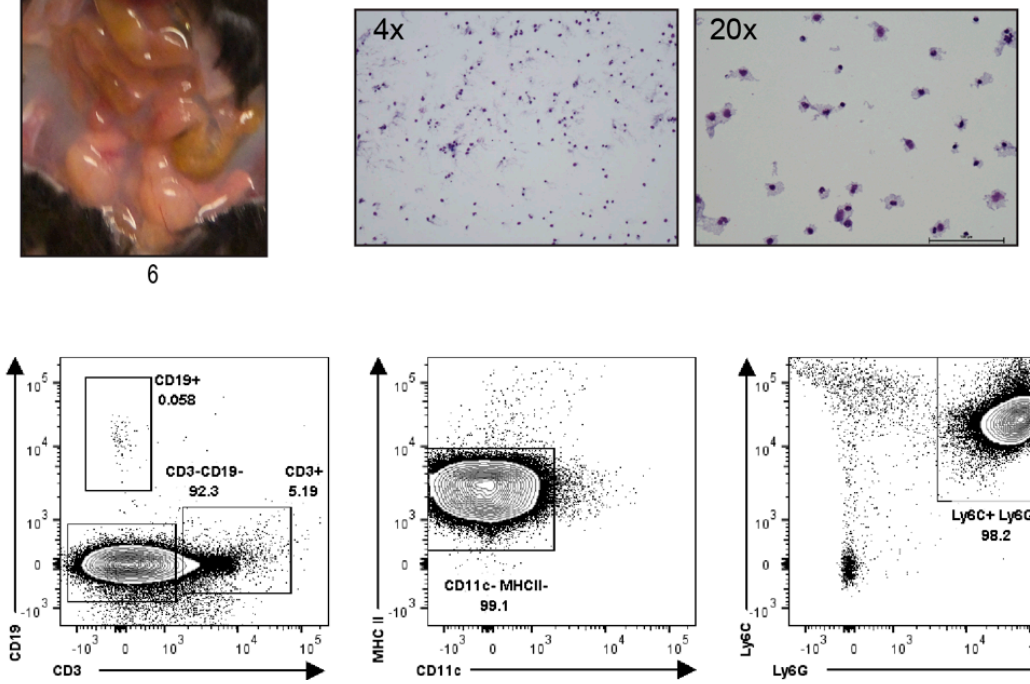

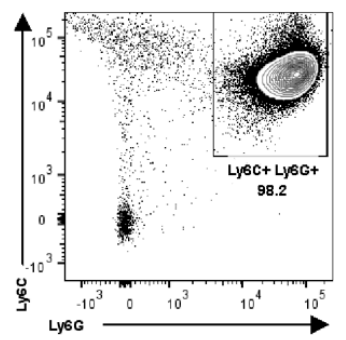

Figure 3. Wright Giemsa stain and flow cytometry analysis of the chylous ascites collected from the peritoneum of 3MC-treated mice on day 6. (A) Accumulation of fluid observed in 3MC-treated WT and Tiparp ${ }^{-/-}$mice. (B) Cellular bodies present in the ascitic fluid. (C) To phenotype the cellular infiltrate, flow cytometry was used and identified the populations to be predominantly neutrophilic. Representative plot of four ascites samples showing a greater neutrophil population (Ly6C+, Ly6G+). Peritoneal fluid was stained directly. Almost all cells in fluid are hematopoietic (CD45.2+) and the majority are innate cell types (CD3- and CD19-). Of these innate cell types, they are not antigen-presenting cells (CD11c- and MHC II-).
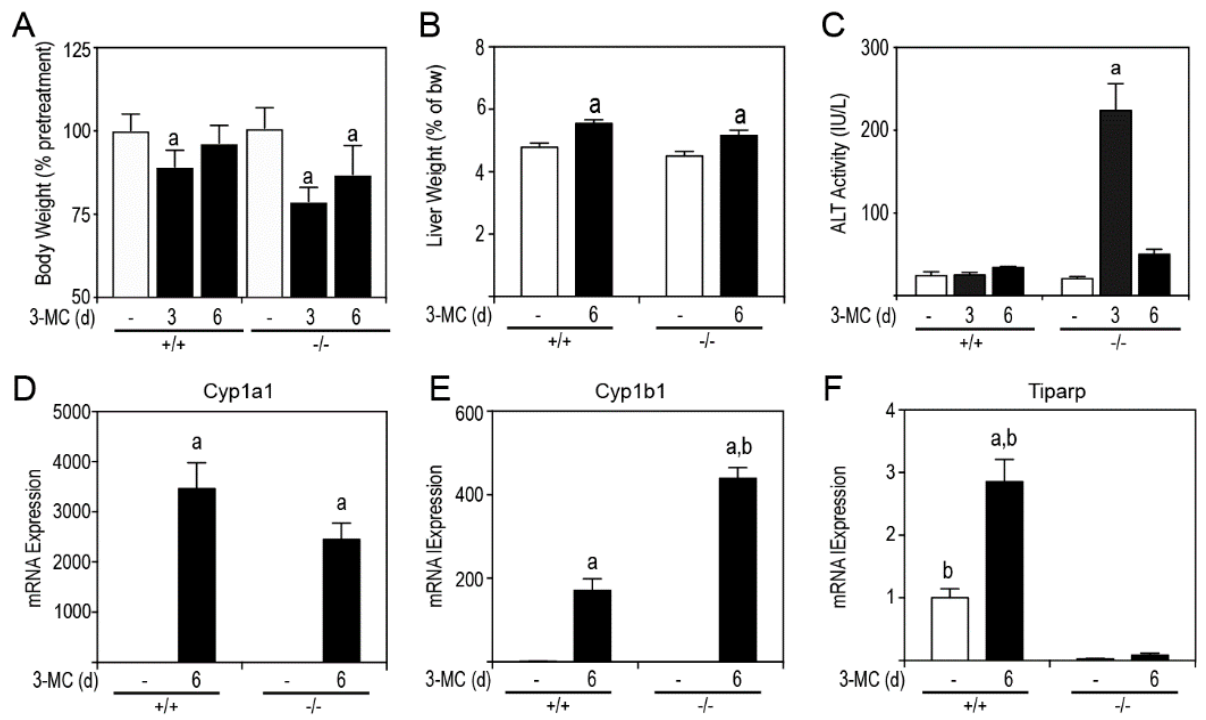

Figure 4. Six-day exposure to $100 \mathrm{mg} / \mathrm{kg} 3 \mathrm{MC}$ causes a transient increase in alanine aminotransferase (ALT) activity and increased hepatic Cyp1b1 but not Cyp1a1 expression levels. (A) Body weight. (B) Liver weight. (C) ALT activity levels. Gene expression levels of Cyp1a1 (D), Cyp1b1 (E), and Tiparp (F). Data represent the mean \pm SEM; $n=3$ for all genes. ${ }^{a} p<0.05$ two-way ANOVA comparison between genotype-matched corn oil- and 3MC-treated mice and ${ }^{\mathrm{b}} p<0.05$ two-way ANOVA comparison between treatment-matched WT and Tiparp ${ }^{-/-}$mice followed by a Tukey's post hoc test. 
The histopathology of the liver was examined to determine if there was any indication of liver toxicity or injury (Figure 5A). Hematoxylin \& Eosin (H\&E) staining of liver sections from corn oil-treated animals displayed an intact architecture with no migratory immune cells from the portal triad or the central vein. WT animals treated with 3MC showed mild microvesicular steatosis as evidenced by vacuolated hepatocytes. Tiparp ${ }^{-1-}$ animals treated with 3MC displayed an increase in the number of resident Küpffer cells in the sinusoids, signifying a mild inflammatory cell infiltration. In support of this increased inflammation, higher levels of the AHR-responsive inflammatory cytokines and chemokines Serpine 1, Il6, Cxcl1, and Cxcl2 were detected in 3MC-treated Tiparp ${ }^{-1}{ }^{-}$mice compared with treatment-matched WT mice (Figure 5B-E). No significant differences in Tnf $\alpha$ and Il-1 $\beta$ levels were observed (Figure 5B-E).

A

3-MC(d)
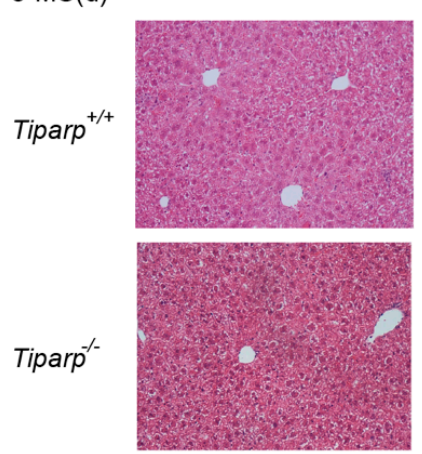

6
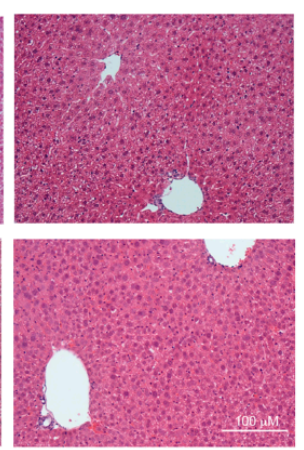

B

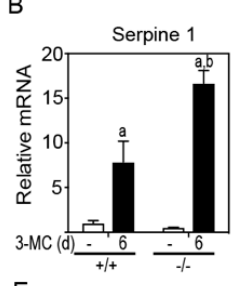

E

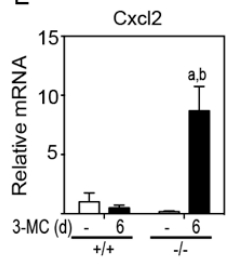

c

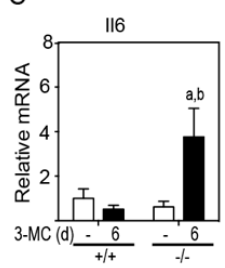

$\mathrm{F}$

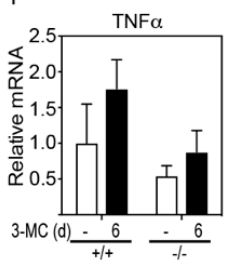

$\mathrm{D}$

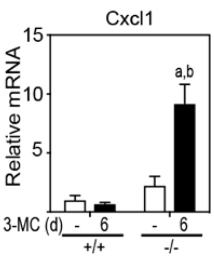

$G$

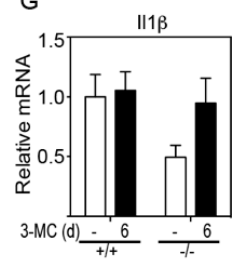

Figure 5. 3MC induces increased inflammation and inflammatory cytokine levels in Tiparp ${ }^{-/-}$compared with WT mice. (A) Hematoxylin \& Eosin (H\&E) staining of the liver lobe at day 6 after corn oil or 3MC treatment. $100 \times$ magnification. Scale bar represents $100 \mu \mathrm{m}$. Gene expression levels of Serpine 1 (PAI-1) (B), Il6 (C), Cxcl1 (D), Cxcl2 (E), Tnf $\alpha(F)$, and IL1 $\beta$ (G). Data represent the mean \pm SEM; $n=3$ for all genes. ${ }^{\mathrm{a}} p<0.05$ two-way ANOVA comparison between genotype-matched corn oil- and 3MC-treated mice and ${ }^{\mathrm{b}} p<0.05$ two-way ANOVA comparison between treatment-matched WT and Tiparp ${ }^{-/-}$mice followed by a Tukey's post hoc test.

Gross liver images from WT corn oil- and 3MC-treated mice, as well as the Tiparp ${ }^{-/}$corn oil-treated animals, displayed a normal liver appearance with a rich red-brownish coloration (Figure 6A). All Tiparp $^{-/-}$mice treated with 3MC were found with white, lobular lesions encapsulating their livers. The source of this white infiltrate appeared to originate from the right lobe, where a white mass was also found in the area. To investigate the involvement of the AHR in hepatic steatosis, Oil Red $\mathrm{O}$ staining was conducted to visualize neutral fats. Both WT and Tiparp ${ }^{-/-}$corn oil-treated mice exhibited normal liver histology (Figure 6B). Conversely, 3MC-treated WT mice had microvesicular fat accumulation around the central vein. However, this was not observed in the 3MC-treated Tiparp ${ }^{-1-}$ mice. No genotype differences in 3MC-induced lipid uptake transporter, $\mathrm{Cd} 36$, levels were observed (Figure 6C). No significant increases in the expression levels of genes involved in lipogenesis (Fasn, Srebp1) and $\beta$-oxidation (Cpt1a) were determined (Figure 6D-F).

Due to the lipid accumulation on internal tissues, perigonadal white adipose tissue (WAT) was removed and weighed at endpoint (Figure 7A). 3MC-treated Tiparp ${ }^{-1-}$ mice had an approximate $60 \%$ reduction in perigonadal WAT levels. The mRNA levels of the AHR target gene, Cypla1, were induced to a higher level in Tiparp $^{-1-}$ mice compared with WT mice (Figure 7B). Tiparp mRNA levels were induced by 3MC treatment in WT mice but not in Tiparp ${ }^{-1}$ mice (Figure 7C). Two lipases-Pnpla2 and Hsl-involved in triglyceride hydrolysis were also studied to examine their expression in WAT and involvement in lipid partitioning. Tiparp ${ }^{-1-}$ mice displayed increased mRNA expression levels of both Pnpla2 and Hsl in WAT compared to corn oil-treated controls or 3MC-treated WT mice (Figure 7D,E). Serum $\beta$-hydroxybutyrate levels were increased in 3MC-treated WT and Tiparp ${ }^{-/-}$mice compared with 
control treated mice (Figure 7F). They were, however, significantly higher in Tiparp ${ }^{-1-}$ mice compared with WT mice, suggesting that the increased lipolysis and resulting free fatty acids are converted into energy and ketone body formation.

A

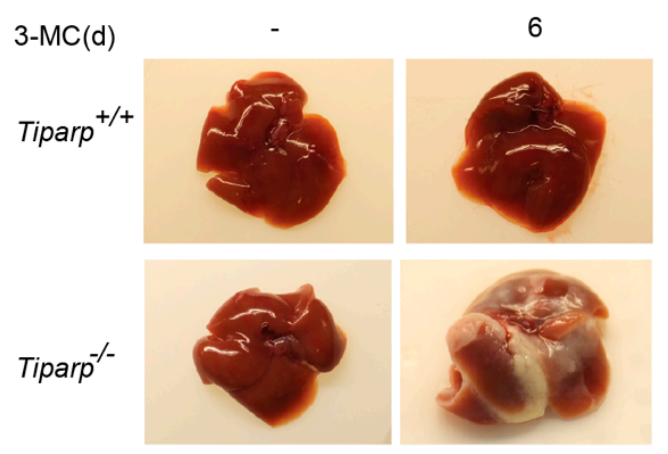

C

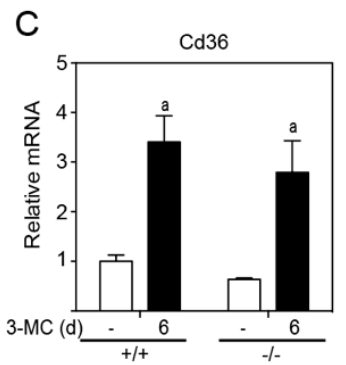

D

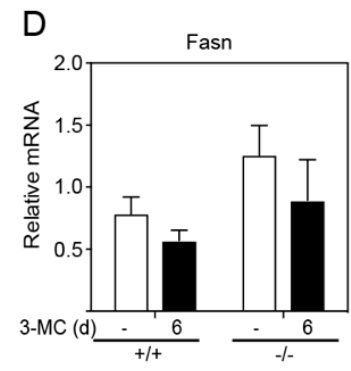

B

$3-\mathrm{MC}(\mathrm{d})$
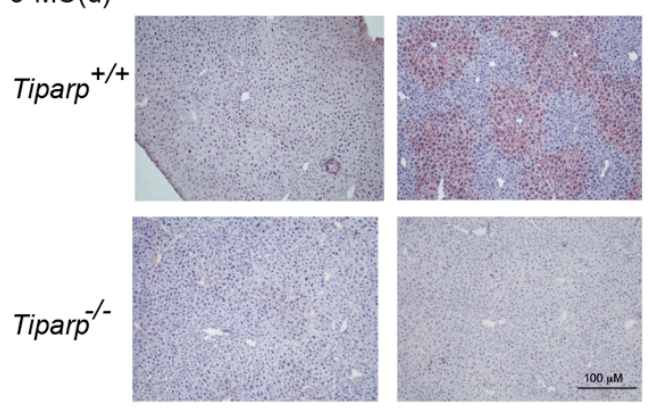

$E$

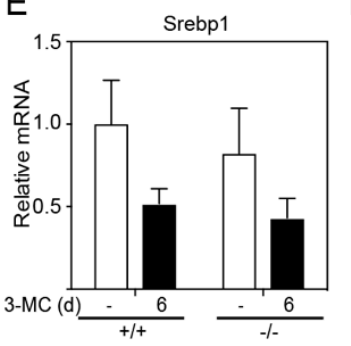

$\mathrm{F}$

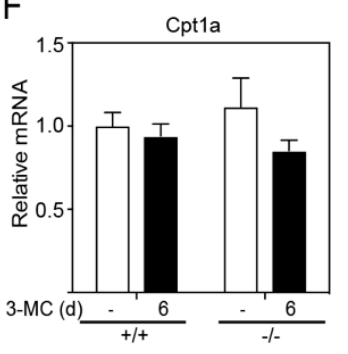

Figure 6. 3MC induces hepatosteatosis in WT but not in Tiparp ${ }^{-1-}$ mice. (A) Gross liver images from corn oil- and 3MC-treated WT and Tiparp ${ }^{-1-}$ mice at day 6. (B) Oil Red O staining was conducted to visualize neutral fats. Gene expression levels of Cd36, (C), Fasn (D), Srebp1 (E), and Cpt1a (F). Data represent the mean \pm SEM of inflammatory genes; $n=3$. ${ }^{a} p<0.05$ two-way ANOVA comparison between genotype-matched corn oil- and 3MC-treated mice followed by a Tukey's post hoc test for multiple comparisons.

A

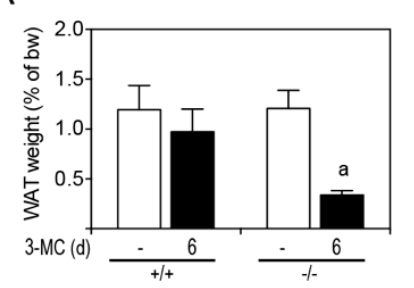

D

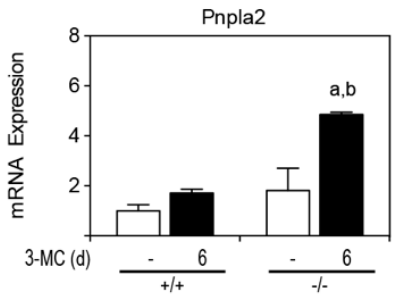

B

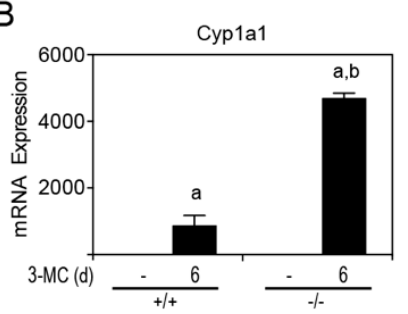

$\mathrm{E}$

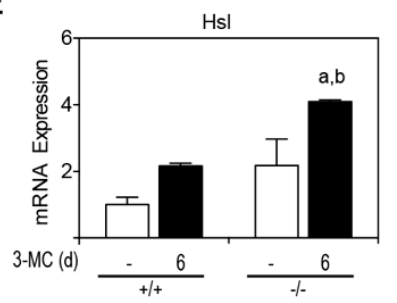

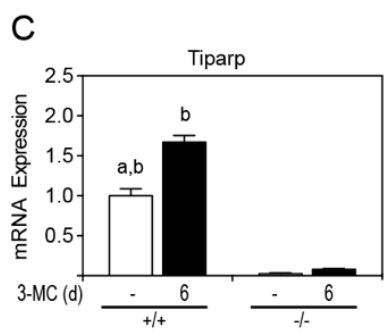

F

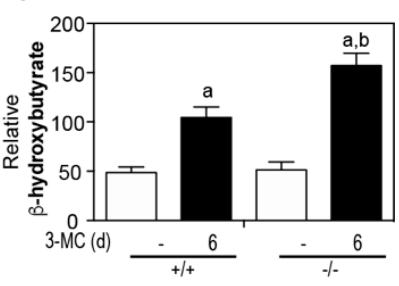

Figure 7. Increased AHR signaling and loss of white adipose tissue (WAT) weight levels in 3MC-treated Tiparp $^{-/}$mice. (A) WAT weight was expressed as a percentage of total body weight on day 6 . The mRNA expression levels of the AHR target genes Cyp1a1 (B), Tiparp (C), Pnpla2 (D), Hsl (E) and serum $\beta$-hydroxybutyrate $(\mathrm{F})$ were measured. Data represent the mean \pm SEM; with an $n=3$ (A). ${ }^{a} p<0.05$ two-way ANOVA comparison between genotype-matched corn oil- and 3MC-treated mice followed by a Tukey's post hoc test for multiple comparisons and ${ }^{\mathrm{b}} p<0.05$ two-way ANOVA comparison between treatment-matched WT and Tiparp ${ }^{-/-}$mice followed by a Tukey's post hoc test. 
To determine if AHR was mediating the 3MC-toxicity, Tiparp $^{-/-}$mice were cotreated with 3MC and the AHR antagonist, $\mathrm{CH} 223191$. Cotreatment with $\mathrm{CH} 223191$ reduced the 3MC dependent increase in Cyp1b1 mRNA levels (Figure 8A), reduced serum ALT activity (Figure 8B) and reduced epididymal WAT loss (Figure 8C). However, CH223191 cotreatment did not prevent 3MC-induced chylous ascites in Tiparp ${ }^{-1-}$ mice, but reduced the severity as indicated by significantly reduced triglyceride levels and increased clarity of the fluid (Table 2).
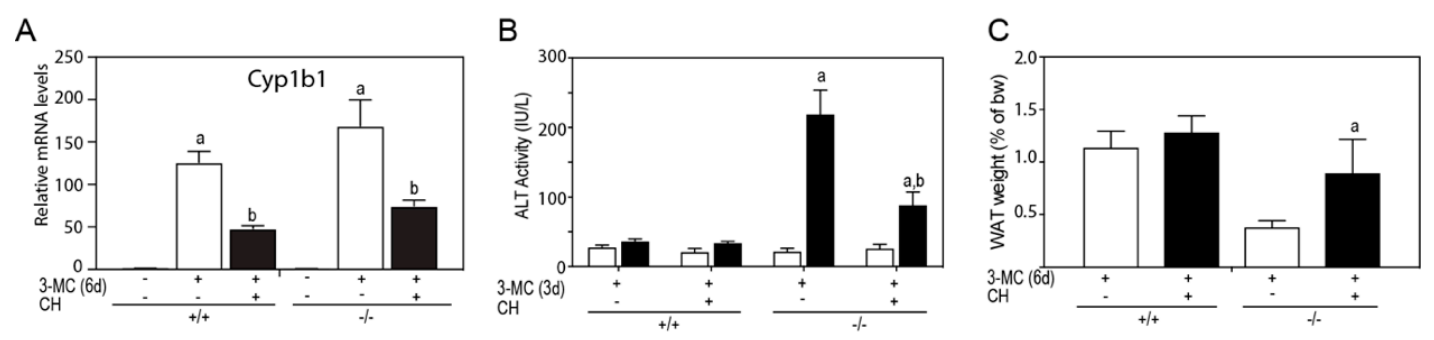

Figure 8. Cotreatment with $\mathrm{CH} 223191$ reduced 3MC-induced alanine aminotransferase (ALT) activity and epididymal adipose tissue loss. WT or Tiparp ${ }^{-/}$mice were cotreated with $100 \mathrm{mg} / \mathrm{kg} 3 \mathrm{MC}$ on day 1 and with $10 \mathrm{mg} / \mathrm{kg} \mathrm{CH223191} \mathrm{(CH)} \mathrm{on} \mathrm{days} 1$ and 3. (A) Hepatic Cyp1b1 mRNA expression levels. (B) ALT activity levels on day 3. (C) WAT weight was expressed as a percentage of total body weight on day 6. Data represent the mean \pm SEM. a $p<0.05$ two-way ANOVA comparison between (A) genotype-matched corn oil- and 3MC-treated mice, (B) genotype-matched day 0 and 3MC-treated day 3 mice, and (C) treatment-matched WT and Tiparp ${ }^{-1-}$ mice on day 6. ${ }^{\mathrm{b}} p<0.05$ two-way ANOVA comparison between WT and Tiparp ${ }^{-1-}$ mice treated with $3 \mathrm{MC}$ and $3 \mathrm{MC}+\mathrm{CH}$ followed by a Tukey's post hoc test.

Table 2. Characteristics of ascites observed after 3MC and CH223191 cotreatment compared to 3MC alone in Tiparp $^{-/-}$mice. Values in parentheses indicate the number of observations over the number of total observations.

\begin{tabular}{ccc}
\hline Measure & Tiparp $^{-/-}$Mice Day 6 & Tiparp $^{-/-}$Mice Day 6 \\
3MC & 3MC + CH223191 \\
\hline Appearance & Milky white and cloudy (4/4) & Clear fluid (1/4) \\
& $560-1670 \mathrm{mg} / \mathrm{dL}$ & white and milky (3/4) \\
Triglyceride Level & (mean: $924 \mathrm{mg} / \mathrm{dL})$ & (mean: $853 \mathrm{mg} / \mathrm{dL})$ \\
Cell Population & Neutrophils & Neutrophils \\
Total Protein & $3.4-4.0 \mathrm{~g} / \mathrm{dL}$ & $3.5-4.0 \mathrm{~g} / \mathrm{dL}$ \\
& $($ mean: $3.6 \mathrm{~g} / \mathrm{dL})$ & $($ mean: $3.7 \mathrm{~g} / \mathrm{dL})$ \\
\hline
\end{tabular}

\section{Discussion}

Here we show that exposure to a high dose of 3MC is lethal to male Tiparp ${ }^{-1-}$ mice, but not to similarly treated WT animals. In previous studies, we reported that whole-body or hepatocyte-specific deletion of Tiparp results in an increased sensitivity to TCDD-induced hepatotoxicity, steatohepatitis and lethal wasting syndrome $[13,24]$. However, unlike TCDD, 3MC-treated Tiparp ${ }^{-1-}$ mice developed a chylous ascites-like condition, with evidence of hepatic inflammation, but without steatosis [25]. Treatment with the AHR inhibitor, CH223191, partially rescued the severity of the chylous ascites, implicating AHR as the mediator of the 3MC-induced toxic outcomes. Our data provide further evidence for the important role of TIPARP in the negative regulation of toxicant-induced AHR activity.

In agreement with previous work, we observed that a single IP injection of $100 \mathrm{mg} / \mathrm{kg} 3 \mathrm{MC}$ resulted in hepatic steatosis in C57BL/6 mice [11]. Hepatic steatosis was, however, not observed in similarly treated Tiparp $^{-/-}$mice. This may have been due to the higher induction of CYP1A1 expression that occurs in the absence of Tiparp, resulting in rapid hepatic 3MC metabolism; thus, preventing hepatic steatosis. In support of this, $\mathrm{Ahrr}^{-/-}$mice show a delayed response to skin carcinogenesis 
caused by benzo[a]pyrene $(\mathrm{B}[a] \mathrm{P})$ due to increased Cyp1a1 levels in skin and more rapid metabolism and clearance of $\mathrm{B}[a] \mathrm{P}$ [26]. However, no increases in hepatic Cyp1a1 levels were reported in liver, lung, and heart, suggesting that the loss of Ahrr expression favors the detoxification of carcinogens via increased Cyp1a1 levels in some but not all tissues. Whether Tiparp ${ }^{-1-}$ mice also show a shift in the metabolism that favors the detoxification of chemical carcinogens has not been determined.

Chylous ascites is defined as a build-up of lymph within the abdomen due to obstruction in the abdominal lymphatic system [27]. Normally, the lymphatic system returns interstitial fluid and proteins to the venous circulation via lymphatic vessels to the lymph nodes. These channels drain into the cisterna chyli at the start of the thoracic duct; however, damage or obstruction to this network can lead to a chyle leak. During such a situation, the lymph fluid becomes milky and viscous due to the conversion of long-chain triglycerides into free fatty acids and monoglycerides, there is an increase in protein content, and an influx of myeloid and lymphoid cell populations [25]. In humans, chylous ascites predominantly contain lymphocytes with a small number of neutrophils [28]. If a predominance of neutrophils is observed, it would be suggestive of peritonitis that may result from an infection [29]. Moreover, AHR activation is known to increase the number of neutrophils recruited to infected tissues, such as lung airways during influenza infection [30]. The chylous ascites observed in the 3MC-treated Tiparp $^{-1-}$ mice was very similar in composition to that observed in humans except for the predominance of neutrophils. Gram staining of the peritoneal fluid was negative for the presence of bacteria (data not shown). Thus, the reason for the neutrophilia in the peritoneal cavity is unknown, but may be in part due to increases in the neutrophil chemoattractants $\mathrm{Cxcl} 1$ and $\mathrm{Cxcl} 2$, which were elevated in livers of 3MC-treated Tiparp ${ }^{-/}$mice. However, their serum or peritoneal fluid levels were not determined in our study. Since chylous ascites was not observed in TCDD-treated Tiparp ${ }^{-/}$mice, the recruitment of neutrophils to the peritoneal cavity is influenced by the nature of the AHR ligand and not a simple result of AHR activation in Tiparp ${ }^{-/-}$mice.

Treatment of Tiparp $^{-/-}$mice with either $10 \mu \mathrm{g} / \mathrm{kg}$ TCDD [13,24] or $100 \mathrm{mg} / \mathrm{kg} 3 \mathrm{MC}$ (the present study) is lethal. However, the effect of each AHR ligand differs with respect to the observed toxicity and the cause of death. TCDD-treated animals display increased sensitivity to hepatotoxicity and wasting syndrome, whereas 3MC-treated animals present with chylous ascites and only mild liver toxicity. Reduced levels of epididymal WAT and increased expression of lipolytic enzymes were consistently observed after treatment with either AHR ligand [13,24]. 3MC-treated Tiparp ${ }^{-1-}$ mice lost significant body weight and epididymal WAT without any reduction in food intake, suggesting that Tiparp loss may affect the efficiency of intestinal fat and or nutrient absorption perhaps due to an obstruction in the lymph. The lack of efficient lipid absorption could explain the increase in lipolysis, which would be needed to provide energy that was not being obtained from the food. This is supported by increased serum $\beta$-hydroxybutyrate levels, suggesting increased energy from $\beta$-oxidation in the liver.

Although this is the first report that 3MC exposure causes chylous ascites, other studies using a variety of transgenic animal models have observed a similar phenotype. In a transgenic mouse model where overexpression of vascular endothelial growth factor (VEGF)-C was induced in adipocytes, chylothorax was observed within seven days of doxycycline treatment in drinking water which led to in overexpression of VEGF-C [31]. Lymphatic vessels in VEGF-C transgenic mice were enlarged and allowed for retrograde flow of milky, triglyceride-rich chyle from the thoracic duct back into the originating lymphatics and, consequently, into the thoracic cavity due to weakened valves and other lymphatic abnormalities promoted by VEGF-C overexpression. The deletion of RASA1, a Ras GTPase-activating protein that negatively regulates lymphatic vessel growth, resulted in a lymphatic vessel disorder characterized by extensive lymphatic vessel hyperplasia, dilation, leakage, and early lethality caused by chylothorax [32]. Patients with a mutation in RASA1 are at a higher risk of developing Parkes-Weber syndrome, which presents itself as a disease with upper and lower extremity lymphedema with some cases of chylothorax and/or chylous ascites [33]. Exposure to TCDD or $3 \mathrm{MC}$ has been demonstrated to upregulate VEGF expression [34]. Moreover, adult Tiparp ${ }^{-1-}$ mice show evidence of vascular defects [35]. Together with the increased sensitivity of Tiparp ${ }^{-1-}$ mice 
to AHR ligands, the activation or downregulation of components in other signaling pathways may lead to the malformation of the lymphatic system, resulting in the accumulation of extravasated fluid. However, whether the accumulation of chylous fluid in the Tiparp ${ }^{-/}$mice is due to the obstruction of the lymphatics or a defect in dietary and endogenous lipid absorption and/or metabolism remains unknown.

In summary, we show that 3MC-treated Tiparp $^{-/-}$mice display an increased sensitivity to 3MC-induced toxicity and lethality, further supporting the role of Tiparp as an important negative regulator of AHR-mediated responses. However, we cannot exclude the possibility that various 3MC metabolites are also involved, since the increased Cyp1a1 levels in 3MC-treated Tiparp ${ }^{-1}$ mice would result in elevated levels of 3MC-derived metabolites. Future studies evaluating alternative routes of administration, lower doses of 3MC, other PAHs and/or AHR ligands, and effects in additional genetically modified mouse models will be needed to determine the etiology of the 3MC-induced chylous ascites in the absence of Tiparp expression.

\section{Materials and Methods}

\subsection{Chemicals and Biological Reagents}

For chemical treatments, a 100-mg vial of 3-methylcholanthrene (3MC) was purchased from Sigma-Aldrich (St. Louis, MO, USA) at an HPLC purity of $>97.5 \%$. Dimethyl sulfoxide (DMSO) and CH223191 were also purchased from Sigma-Aldrich. One-hundred percent pure corn oil (CO) was purchased from a local grocer. A $10 \mathrm{mg} / \mathrm{mL}$ stock of $3 \mathrm{MC}$ was made and this solution was made fresh before injection and disposed of after 30 days. Liver sections for H\&E staining were preserved in neutral buffered 10\% formalin solution (Sigma-Aldrich), and liver sections for Oil Red O were suspended in VWR ${ }^{\circledR}$ Clear Frozen Section Compound (Radnor, PA, USA) to embed tissues for cryosectioning. The Infinity ${ }^{\mathrm{TM}}$ ALT Liquid Stable Reagent was purchased from Fisher Diagnostics (Middletown, VA, USA) for use in the in vitro determination of ALT activity in mouse serum.

\subsection{Animals}

TiparpGt(ROSA)79Sor mutant mice (stock number: 007206) were purchased from Jackson Laboratories (Bar Harbor, ME, USA) and have been previously described [13,35]. The animal colony was maintained by breeding heterozygotes. Only WT $\left(\right.$ Tiparp $\left.^{+/+}\right)$and Tiparp ${ }^{-1-}$ mice were used in experiments. Animals were housed in the Division of Comparative Medicine at the University of Toronto (Toronto, ON, Canada). The temperature was constant at $21^{\circ} \mathrm{C}$; a maintained light-dark cycle (12 $\mathrm{h}$ and $12 \mathrm{~h}$ ); humidity within the facility was controlled; and standard rodent chow and sterile water were provided ad libitum. All procedures and experiments conducted were in accordance with the principles set by the Canadian Council on Animal Care guidelines and approved by the Local Animal Care Committee (protocol \# 20010338) on the 9th of September 2014 at the University of Toronto.

\subsection{MC and $\mathrm{CH} 223191$ Treatment}

Seven-to-nine week old male Tiparp ${ }^{+/+}$and Tiparp $^{-/-}$mice were given a single intraperitoneal injection of $100 \mathrm{mg} / \mathrm{kg}$ body weight of $3 \mathrm{MC}$ dissolved in corn oil. Control (corn oil) mice received an equivalent volume of corn oil corrected for body weight. For 3MC and the AHR antagonist CH223191 cotreatment studies, mice were injected with $100 \mathrm{mg} / \mathrm{kg} 3 \mathrm{MC}$ and $10 \mathrm{mg} / \mathrm{kg} \mathrm{CH} 223191$ dissolved in DMSO or an equivalent volume of DMSO as control. The animals received a second IP injection of $\mathrm{CH}$ or DMSO on day 3. Solutions were heated to $37^{\circ} \mathrm{C}$ and vortexed to ensure solubilization of the compound prior to treatment. Mice were monitored daily and proper personal protective equipment was implemented for the handling of 3MC-treated animals. If considered endpoints were met at any moment during the experiment, humane intervention was implemented to prevent or relieve unnecessary pain and distress. Suggested endpoints include body weight loss exceeding $20 \%$ of normal body weight as measured on day 0 , severe lethargy and reluctance to move when provoked, 
hunched or abnormal posture, severe dehydration or malnutrition, and signs of severe discomfort. If these ailments could not be alleviated through preventative measures, then the mice were humanely euthanized. Animals were supplied with standard chow (Teklad Global Diet ${ }^{\circledR} 2018 ; 18 \%$ protein, 6\% fat) in the form of pelleted food from Harlan Laboratories (Indianapolis, IN, USA).

\subsection{Body and Food Weight Measurements}

Mice and food pellets were weighed daily in the morning and values were recorded throughout the study. Body weight was taken after stabilization of weight fluctuation by the scale. For food intake measurements, $100 \mathrm{~g}$ of intact food pellets were placed on the top wire feeder and weighed on Day-1 for the calculation of the baseline value (day 0). All pellets on top of the wire feeder as well as any residual pieces on the cage floor were accounted for in the daily food intake measurement. Measurements recorded on each subsequent day were subtracted from the previous day's recorded value to provide the daily food intake value. Body weight and food intake values were normalized to baseline and graphed as an increase or decrease from day 0.

\subsection{Blood Collection and ALT analysis}

Blood was collected from the saphenous vein of the hind leg. Approximately $100 \mu \mathrm{L}$ of blood was collected in a Microvette ${ }^{\circledR} 200$ Z-Gel tube, and this was conducted on day 0 (before treatment for baseline values), day 3 , and day 6 . Blood samples were placed at room temperature for a minimum of $30 \mathrm{~min}$ for coagulation. The sample can then be centrifuged at 10,000 rpm for $5 \mathrm{~min}$ to separate the serum, which was subsequently collected and stored at $-80^{\circ} \mathrm{C}$ until ALT analysis. The Infinity ${ }^{\mathrm{TM}}$ ALT (GPT) Liquid Stable Reagent (Fisher Diagnostics) was warmed to $37^{\circ} \mathrm{C}$ for optimal assay conditions. Samples were processed in duplicates. Immediately before assay measurements, $160 \mu \mathrm{L}$ of the ALT reagent was aliquoted into each well. The constant temperature was set at $37^{\circ} \mathrm{C}$ within the BioTek Synergy ${ }^{\mathrm{TM}}$ MX multi-mode microplate reader (BioTek Instruments) and kinetic measurements were taken at an absorbance of $340 \mathrm{~nm}$ each minute for a total duration of $15 \mathrm{~min}$. Activity levels were adjusted with the recommended factor. Values were then plotted against time and the average of the two slopes was obtained.

\section{6. $\beta$-Hydroxybutyrate Levels}

$\beta$-hydroxybutyrate levels were measured using an assay kit (Sigma-Aldrich). A serum sample volume of $10 \mu \mathrm{L}$ was used and was directly added onto a 96-well plate. The assay was performed according to the supplier's specifications and preparation instructions. Absorbance was measured at $450 \mathrm{~nm}$.

\subsection{RNA Extraction and Isolation}

Mice were humanely euthanized by cervical dislocation and the whole liver was washed in ice-cold PBS, dried quickly on absorbent paper, and recorded for tissue weight. Epididymal WAT was removed from the perigonadal region washed in ice-cold PBS and weighed. All tissues were flash-frozen immediately in liquid nitrogen after recording tissue weights. Collected fluid samples were prepared on microscope slides before storing at $-80^{\circ} \mathrm{C}$. For RNA isolation from liver, approximately $50 \mathrm{mg}$ of frozen liver was homogenized in $500 \mu \mathrm{L}$ of TRIzol ${ }^{\circledR}$ reagent. Samples were incubated at room temperature to allow for the complete dissociation between complexes before the addition of chloroform. The samples were vigorously vortexed and centrifuged at $13,000 \mathrm{rpm}$ for $15 \mathrm{~min}$ at $4{ }^{\circ} \mathrm{C}$ for phase separation. The RNA, located in the upper aqueous phase, was added with $70 \%$ ethanol of equal volume. The lysate was thoroughly mixed and transferred into RNA binding columns supplied by the Aurum ${ }^{\mathrm{TM}}$ Total RNA Mini Kit. Once the RNA was eluted, these tubes were placed directly on ice. RNA concentration, purity, and quality were measured using the spectrophotometer at a 40 -fold dilution in water. Extracted liver RNA samples were adjusted to $50 \mathrm{ng} / \mu \mathrm{L}$ with the addition of DNase/RNase-free distilled water. 


\section{8. cDNA Synthesis and Gene Expression Analyses}

For the synthesis of cDNA from RNA, $10 \mu \mathrm{L}$ of $500 \mathrm{ng}$ normalized liver was reverse transcribed using SuperScript ${ }^{\circledR} \mathrm{III}$ and its components. The reaction mix consisted of $4 \mu \mathrm{L} 5 \times$ First Strand buffer, $0.1 \mathrm{mM}$ DTT, $50 \mathrm{mM}$ random hexamers, $10 \mu \mathrm{M}$ dNTP mixture, distilled water, and SuperScript ${ }^{\circledR} \mathrm{III}$ in a total reaction volume of $20 \mu \mathrm{L}$ per sample. Using the MJ Cycler Software 2.0 (Bio-Rad) on the Bio-Rad Chromo4 ${ }^{\mathrm{TM}}$ DyadDisciple ${ }^{\mathrm{TM}}$, the cDNA synthesis reaction involved an initial $1 \mathrm{~h}$ incubation at $50^{\circ} \mathrm{C}$ followed by a $15 \mathrm{~min}$ at $70^{\circ} \mathrm{C}$ to inactivate the enzyme. The synthesized reaction was then diluted with DNase/RNase-free distilled water. The qPCR reaction was prepared using SsoFast EvaGreen ${ }^{\circledR}$ SYBR Supermix, and $10 \mu \mathrm{M}$ forward and reverse primer verified with NCBI Primer-BLAST (Bethesda, MD, USA). Technical duplicates were performed for each target gene transcript and normalized to the tata binding protein (Tbp) mRNA content. Reactions were performed on the Bio-Rad Chromo4 ${ }^{\mathrm{TM}}$ DyadDisciple ${ }^{\mathrm{TM}}$ with the following conditions; $95^{\circ} \mathrm{C}$ for $3 \mathrm{~min}$ and 45 cycles of $95{ }^{\circ} \mathrm{C}$ for $5 \mathrm{~s}$ for denaturation and $60^{\circ} \mathrm{C}$ for $20 \mathrm{~s}$. Data were analyzed using the Opticon Monitor ${ }^{\mathrm{TM}} 3$ software (Bio-Rad) and fold changes were computed by the comparative cycle threshold $(\Delta \Delta \mathrm{CT})$ method and normalized to corn oil-treated WT controls.

\subsection{Tissue Histology}

To prepare slides for Hematoxylin \& Eosin (H\&E) staining, liver sections obtained from the animal dissections were freshly fixed in neutral buffered $10 \%$ formalin solution before processing and paraffin embedding. In this procedure, the liver was sectioned into 5 - $\mu \mathrm{m}$-thick segments. To prepare slides for Oil Red O staining, liver samples were suspended in VWR ${ }^{\circledR}$ Clear Frozen Section Compound and flash-frozen in liquid nitrogen as previously described [13]. Sections of $5-\mu \mathrm{m}$-thick tissue-embedded ribbons were sliced using a cryostat and adhered onto a glass slide. The aforementioned procedures were services provided at Princess Margaret Hospital (Toronto, ON, Canada) of the University Health Network. For each slide, representative images of the cell population were obtained at $40 \times, 100 \times$, and $200 \times$ magnification.

\subsection{Wright Giemsa Stain}

Peritoneal ascites was suspected from the observable distension of the abdomen, the peritoneum was carefully slit for the insertion of a 1cc Luer-slip syringe and withdrawal of the fluid for inspection and analysis. An aliquot of the sample was smeared as a thin film across a microscope slide using aseptic techniques over an open flame. The sample was allowed to air-dry before fixing in $100 \%$ methanol. The fixed sample was flooded with modified Accustain ${ }^{\circledR}$ Wright Giemsa stain and an equal volume of distilled water was added to the stain. For visualization, Wright Giemsa-stained slides were imaged using a brightfield microscope and Nikon NIS-Elements Viewer imaging software. For each slide, representative images of the cell population were obtained at $40 \times, 100 \times$, and $200 \times$ magnification.

\subsection{Flow Cytometry}

Peritoneal ascites were isolated and stained directly using fixable viability dye in eF506 (eBioscience, San Diego, USA) and for surface markers using the following antibodies; anti-mouse CD45.2 in FITC (clone: 104; eBioscience), anti-mouse CD3 $\varepsilon$ in PE-Cy7 (clone: 145-2C11; eBioscience), anti-mouse CD19 in BV605 (clone: 6D5; BioLegend, San Diego, USA), anti-mouse MHC class II in e450 (clone: AF6-120.1; eBioscience), anti-mouse CD11c in AF700 (clone: N418; eBioscience), anti-mouse Ly6C in PerCP-Cy5.5 (clone: HK1.4; eBioscience), and anti-mouse Ly6G in APC (RB6-8C5; eBioscience). Samples were analyzed using LSRFortessa (BD Biosciences, San Jose, USA) and FlowJo (TreeStar Inc., Ashland, USA) software. 


\subsection{Statistical Analysis}

Daily body weight and food intake measures are expressed as the mean \pm standard error of the mean (SEM) across all animals and analyzed by repeated measures two-way analysis of variance (ANOVA) with a Tukey's post hoc statistical test for multiple comparisons between day-matched mice. A log-rank (Mantel-Cox) test was used in the survival curve analyses to determine significance $(p<0.05)$ between groups. In all other results, a two-way analysis of variance (ANOVA) followed by Tukey's multiple comparisons test was used to determine statistical significance $(p<0.05)$. All data were graphed and analyzed using GraphPad Prism 6 statistical software (San Diego, CA, USA) using grouped measures.

Author Contributions: T.E.C., D.B., S.A., D.H., A.G., L.T., A.C.Z., D.M.G., and J.M. carried out most of the experiments and analyzed the data. T.H.W., D.M.G., and J.M. designed and supervised the study. J.M. wrote the manuscript with critical input from all coauthors.

Funding: This work was supported by Canadian Institutes of Health Research (CIHR) operating grants (MOP-494265 and MOP-125919), the Johan Throne Holst Foundation, and by an unrestricted research grant from the DOW Chemical Company to J.M. The funding sources had no role in the design of this study and they did not have any role during in its execution, analyses, interpretation of the data, or decision to submit results.

Acknowledgments: The authors acknowledge Otto Sanchez from University of Ontario Institute of Technology for his help with the histological analyses and Andrew Elia and Lily Zhou from CFIBCR Histology/Microscopy Core, Princess Margaret Cancer Center, University Health Network, Toronto for preparing and staining the histological slides.

Conflicts of Interest: The authors declare no conflicts of interest. The funders had no role in the design of the study; in the collection, analyses, or interpretation of data; in the writing of the manuscript, or in the decision to publish the results.

\section{Abbreviations}

$\begin{array}{ll}\text { 3MC } & \text { 3-methylcholanthrene } \\ \text { AHR } & \text { aryl hydrocarbon receptor } \\ \text { AHRE } & \text { AHR response element } \\ \text { ARNT } & \text { AHR nuclear translocator } \\ \text { ARTD } & \text { ADP-ribosyltransferase diphtheria toxin-like } \\ \text { B[a]P } & \text { benzo[a]pyrene } \\ \text { CYP1A1 } & \text { cytochrome P450 1A1 } \\ \text { IP } & \text { intraperitoneal } \\ \text { PAH } & \text { polycyclic aromatic hydrocarbon } \\ \text { PARP } & \text { poly-ADP-ribose polymerase } \\ \text { TCDD } & \text { 2,3,7,8-tetrachlorodibenzo- } p \text {-dioxin } \\ \text { TIPARP } & \text { TCDD-inducible poly-ADP-ribose polymerase } \\ \text { WAT } & \text { white adipose tissue }\end{array}$

\section{References}

1. Hankinson, O. The aryl hydrocarbon receptor complex. Annu. Rev. Pharm. Toxicol. 1995, 35, 307-340. [CrossRef] [PubMed]

2. Ma, Q.; Baldwin, K.T.; Renzelli, A.J.; McDaniel, A.; Dong, L. TCDD-inducible poly(ADP-ribose) polymerase: A novel response to 2,3,7,8-tetrachlorodibenzo-p-dioxin. Biochem. Biophys. Res. Commun. 2001, 289, 499-506. [CrossRef] [PubMed]

3. Shimada, T.; Fujii-Kuriyama, Y. Metabolic activation of polycyclic aromatic hydrocarbons to carcinogens by cytochromes P450 1A1 and 1B1. Cancer Sci. 2004, 95, 1-6. [CrossRef] [PubMed]

4. Matthews, J. Aryl Hydrocarbon Receptor (AhR) Targeted by Xenobiotic Compounds and Dietary Phytochemicals. In Hormone-Disruptive Chemical Contaminants in Food, Pongratz, I., Ed; RSC Issues in Toxicology Series: Cambridge, UK, 2013.

5. Larsson, B.K.; Sahlberg, G.P.; Eriksson, A.T.; Busk, L.A. Polycyclic aromatic hydrocarbons in grilled food. J. Agric. Food Chem. 1983, 31, 867-873. [CrossRef] [PubMed] 
6. Fertuck, K.C.; Kumar, S.; Sikka, H.C.; Matthews, J.B.; Zacharewski, T.R. Interaction of PAH-related compounds with the alpha and beta isoforms of the estrogen receptor. Toxicol. Lett. 2001, 121, 167-177. [CrossRef]

7. Hyzd'alova, M.; Pivnicka, J.; Zapletal, O.; Vazquez-Gomez, G.; Matthews, J.; Neca, J.; Pencikova, K.; Machala, M.; Vondracek, J. Aryl Hydrocarbon Receptor-Dependent Metabolism Plays a Significant Role in Estrogen-Like Effects of Polycyclic Aromatic Hydrocarbons on Cell Proliferation. Toxicol. Sci. 2018, 165, 447-461. [CrossRef]

8. Rhon-Calderon, E.A.; Toro, C.A.; Lomniczi, A.; Galarza, R.A.; Faletti, A.G. Changes in the expression of genes involved in the ovarian function of rats caused by daily exposure to 3-methylcholanthrene and their prevention by alpha-naphthoflavone. Arch. Toxicol. 2018, 92, 907-919. [CrossRef]

9. Bresnick, E.; Foldes, R.; Hines, R.N. Induction of cytochrome P450 by xenobiotics. Pharm. Rev. 1984, 36, 43S-51S.

10. Helle, J.; Keiler, A.M.; Zierau, O.; Dorfelt, P.; Vollmer, G.; Lehmann, L.; Chittur, S.V.; Tenniswood, M.; Welsh, J.; Kretzschmar, G. Effects of the aryl hydrocarbon receptor agonist 3-methylcholanthrene on the 17beta-estradiol regulated mRNA transcriptome of the rat uterus. J. Steroid. Biochem. Mol. Biol. 2017, 171, 133-143. [CrossRef]

11. Kawano, Y.; Nishiumi, S.; Tanaka, S.; Nobutani, K.; Miki, A.; Yano, Y.; Seo, Y.; Kutsumi, H.; Ashida, H.; Azuma, T.; et al. Activation of the aryl hydrocarbon receptor induces hepatic steatosis via the upregulation of fatty acid transport. Arch. Biochem. Biophys. 2010, 504, 221-227. [CrossRef]

12. MacPherson, L.; Tamblyn, L.; Rajendra, S.; Bralha, F.; McPherson, J.P.; Matthews, J. 2,3,7,8-tetrachlorodibenzo- $p$-dioxin poly(ADP-ribose) polymerase (TiPARP, ARTD14) is a mono-ADP-ribosyltransferase and repressor of aryl hydrocarbon receptor transactivation. Nucleic Acids Res. 2013, 41, 1604-1621. [CrossRef]

13. Ahmed, S.; Bott, D.; Gomez, A.; Tamblyn, L.; Rasheed, A.; MacPherson, L.; Sugamori, K.S.; Cho, T.; Yang, Y.; Grant, D.M.; et al. Loss of the Mono-ADP-Ribosyltransferase, TIPARP, Increases Sensitivity to Dioxin-Induced Steatohepatitis and Lethality. J. Biol. Chem. 2015, 290, 16824-16840. [CrossRef]

14. Bindesboll, C.; Tan, S.; Bott, D.; Cho, T.; Tamblyn, L.; MacPherson, L.; Gronning-Wang, L.M.; Nebb, H.I.; Matthews, J. TCDD-inducible poly-ADP-ribose polymerase (TIPARP/PARP7) mono-ADP-ribosylates and coactivates liver X receptors. Biochem. J. 2016, 473, 899-910. [CrossRef]

15. Luscher, B.; Butepage, M.; Eckei, L.; Krieg, S.; Verheugd, P.; Shilton, B.H. ADP-Ribosylation, a Multifaceted Posttranslational Modification Involved in the Control of Cell Physiology in Health and Disease. Chem. Rev. 2018, 118, 1092-1136. [CrossRef]

16. Vyas, S.; Chesarone-Cataldo, M.; Todorova, T.; Huang, Y.H.; Chang, P. A systematic analysis of the PARP protein family identifies new functions critical for cell physiology. Nat. Commun. 2013, 4, 2240. [CrossRef]

17. Gomez, A.; Bindesboll, C.; Satheesh, S.V.; Grimaldi, G.; MacPherson, L.; Hutin, D.; Ahmed, S.; Tamblyn, L.; Cho, T.; Nebb, H.I.; et al. Characterization of TCDD-Inducible Poly-ADP-Ribose Polymerase (TIPARP/ARTD14) Catalytic Activity. Biochem. J. 2018, 475, 3827-3846. [CrossRef]

18. Han, B.; Zhang, Y.; Zhang, Y.; Bai, Y.; Chen, X.; Huang, R.; Wu, F.; Leng, S.; Chao, J.; Zhang, J.H.; et al. Novel insight into circular RNA HECTD1 in astrocyte activation via autophagy by targeting MIR142-TIPARP: Implications for cerebral ischemic stroke. Autophagy 2018, 14, 1164-1184. [CrossRef]

19. Yamada, T.; Horimoto, H.; Kameyama, T.; Hayakawa, S.; Yamato, H.; Dazai, M.; Takada, A.; Kida, H.; Bott, D.; Zhou, A.C.; et al. Constitutive aryl hydrocarbon receptor signaling constrains type I interferon-mediated antiviral innate defense. Nat. Immunol. 2016, 17, 687-694. [CrossRef]

20. Kozaki, T.; Komano, J.; Kanbayashi, D.; Takahama, M.; Misawa, T.; Satoh, T.; Takeuchi, O.; Kawai, T.; Shimizu, S.; Matsuura, Y.; et al. Mitochondrial damage elicits a TCDD-inducible poly(ADP-ribose) polymerase-mediated antiviral response. Proc. Natl. Acad. Sci. USA 2017, 114, 2681-2686. [CrossRef]

21. Chen, W.V.; Delrow, J.; Corrin, P.D.; Frazier, J.P.; Soriano, P. Identification and validation of PDGF transcriptional targets by microarray-coupled gene-trap mutagenesis. Nat. Genet. 2004, 36, 304-312. [CrossRef]

22. Atasheva, S.; Akhrymuk, M.; Frolova, E.I.; Frolov, I. New PARP gene with an anti-alphavirus function. J. Virol. 2012, 86, 8147-8160. [CrossRef]

23. Sasse, S.K.; Mailloux, C.M.; Barczak, A.J.; Wang, Q.; Altonsy, M.O.; Jain, M.K.; Haldar, S.M.; Gerber, A.N. The glucocorticoid receptor and KLF15 regulate gene expression dynamics and integrate signals through feed-forward circuitry. Mol. Cell. Biol. 2013, 33, 2104-2115. [CrossRef] 
24. Hutin, D.; Tamblyn, L.; Gomez, A.; Grimaldi, G.; Soedling, H.; Cho, T.; Ahmed, S.; Lucas, C.; Chakravarthi, K.; Grant, D.M.; et al. Hepatocyte-specific deletion of TIPARP, a negative regulator of the aryl hydrocarbon receptor, is sufficient to increase sensitivity to dioxin-induced wasting syndrome. Toxicol. Sci. 2018, 165, 347-360. [CrossRef]

25. Cárdenas, A.; Chopra, S. Chylous ascites. Am. J. Gastroenterol. 2002, 97, 1896-1900. [CrossRef]

26. Hosoya, T.; Harada, N.; Mimura, J.; Motohashi, H.; Takahashi, S.; Nakajima, O.; Morita, M.; Kawauchi, S.; Yamamoto, M.; Fujii-Kuriyama, Y. Inducibility of cytochrome P450 1A1 and chemical carcinogenesis by benzo[a]pyrene in AhR repressor-deficient mice. Biochem. Biophys. Res. Commun. 2008, 365, 562-567. [CrossRef]

27. Jardinet, T.; Verbeke, L.; Bonne, L.; Maleux, G. Therapeutic intranodal lymphangiography for refractory chylous ascites complicating acute necrotic pancreatitis. J. Gastrointestin. Liver Dis. 2018, 27, 195-197.

28. Santos, M.A.; Bose, P.S.; Maher, S.; Desai, M. Chylous Ascites: An Unusual Complication of Necrotizing Pancreatitis. Am. J. Med. 2017, 130, e151-e152. [CrossRef]

29. Huang, L.L.; Xia, H.H.; Zhu, S.L. Ascitic Fluid Analysis in the Differential Diagnosis of Ascites: Focus on Cirrhotic Ascites. J. Clin. Transl. Hepatol. 2014, 2, 58-64.

30. Stevens, E.A.; Mezrich, J.D.; Bradfield, C.A. The aryl hydrocarbon receptor: A perspective on potential roles in the immune system. Immunology 2009, 127, 299-311. [CrossRef]

31. Nitschke, M.; Bell, A.; Karaman, S.; Amouzgar, M.; Rutkowski, J.M.; Scherer, P.E.; Alitalo, K.; McDonald, D.M. Retrograde Lymph Flow Leads to Chylothorax in Transgenic Mice with Lymphatic Malformations. Am. J. Pathol. 2017, 187, 1984-1997. [CrossRef]

32. Lapinski, P.E.; Kwon, S.; Lubeck, B.A.; Wilkinson, J.E.; Srinivasan, R.S.; Sevick-Muraca, E.; King, P.D. RASA1 maintains the lymphatic vasculature in a quiescent functional state in mice. J. Clin. Invest. 2012, 122, 733-747. [CrossRef] [PubMed]

33. Burrows, P.E.; Gonzalez-Garay, M.L.; Rasmussen, J.C.; Aldrich, M.B.; Guilliod, R.; Maus, E.A.; Fife, C.E.; Kwon, S.; Lapinski, P.E.; King, P.D.; et al. Lymphatic abnormalities are associated with RASA1 gene mutations in mouse and man. Proc. Natl. Acad. Sci. USA 2013, 110, 8621-8626. [CrossRef] [PubMed]

34. Terashima, J.; Tachikawa, C.; Kudo, K.; Habano, W.; Ozawa, S. An aryl hydrocarbon receptor induces VEGF expression through ATF4 under glucose deprivation in HepG2. BMC Mol. Biol. 2013, 14, 27. [CrossRef] [PubMed]

35. Schmahl, J.; Raymond, C.S.; Soriano, P. PDGF signaling specificity is mediated through multiple immediate early genes. Nat. Genet. 2007, 39, 52-60. [CrossRef] [PubMed] 